\title{
438 - Nigerian women are more susceptible to the impact of diabetes-and-dementia: State- of-art, Future perspectives and Directions
}

Giménez-Llort $L^{1,2,6}$, Oghagbon $E K^{3,4}$, Dogo $F^{4}$, Ogiator $M^{5}$, Prieto-Pino $J^{1,2}$

${ }^{1}$ Department of Psychiatry and Forensic Medicine, Faculty of Medicine, Universitat Autònoma de Barcelona, Barcelona, Spain

${ }^{2}$ Institut de Neurociències, Universitat Autònoma de Barcelona, Barcelona, Spain

${ }^{3}$ Department of Chemical Pathology, Faculty of Basic \& Allied Medical Sciences, College of Health Sciences, Benue State University, Makurdi, Nigeria.

${ }^{4}$ Department of Chemical Pathology, Benue State University Teaching Hospital, Makurdi, Nigeria

${ }^{5}$ Department. of Internal Medicine, Benue State University Teaching Hospital, Makurdi, Nigeria

${ }^{6}$ Envellir bé - Saber Envejecer - Healthy Aging Charity Organization, Sant Quirze del Vallès, Barcelona, Spain

Among the preventable complications of diseases that require urgent effective health literacy programs in sub-Saharan Africa, crosstalk between diabetes and dementia stands out for women's health. Type 2 diabetes mellitus (DM2) in midlife is a recognised risk factor for dementia. This crosstalk is more significant in persons of African ancestry. Globally, the prevalence of DM will increase dramatically in the next few years with $75 \%$ of cases living in low-to-middle-income countries. Some major risk factors for DM2 accelerates the development of dementia in Africa-Americans, thus leading to higher prevalence of dementia compared to Caucasians. It is known that $58 \%$ of the global 46.8 million dementia subjects lives in economically developing countries. This proportion may reach $63 \%$ and $68 \%$ in 12 and 32 years' time, respectively. Females are 1.5 times likely to develop dementia, but sub-Saharan Africa women have a disproportionately two-to-eight fold increased dementia risk. In the eye of this storm is Nigeria which is home to the highest number of diabetics in Africa. Diabetes prevalence in the country is rising parallel to increased incidence of obesity, hypertension and rising population age. The socioeconomic impact of increasing prevalence of DM2 and dementia will be unsustainable for Nigeria healthcare system, given the experiences in developed economies. This study analyses the current situation of women's health in Nigeria, and explore future policy directions. The complex interplay of factors involved in the DM2-dementia crosstalk in Nigerian women include those due to biological processes (metabolic syndrome, vascular damage, inflammation, oxidative stress, insulin resistance and anaemia), nutritional habits and sedentary lifestyles. Other factors that predisposes Nigerian diabetic women to dementia are, restricted resources, lack of visibility and poor health management. They add up to increase the burden of disease in the Nigerian woman, irrespective of age. We advise urgent implementation of heath policies and actions that will increase ratio of mental health professionals / number of patients, especially in rural areas and the establishment of proactive primary healthcare centres. Importantly, interventions targeting adolescents and adult women, and others specific to mother-child interactions, are strongly needed in Nigeria and the sub-region for mitigating dementia in women. 